Supporting Information for the Paper

\title{
Bidirectional Phase Transformation of Supramolecular Networks Using Two Molecular Signals
}

Daling Cui, Cheng-Hao Liu, Federico Rosei*, and Dmitrii F. Perepichka*

Email: Dmitrii.perepichka@mcgill.ca; federico.rosei@inrs.ca

\section{TMTPT synthesis and characterization}

TMTPT was synthesized according to a modified literature procedure. ${ }^{1}$ In a $100 \mathrm{~mL}$ round bottom flask, $1.45 \mathrm{~g}$ (148 mmol) of 3-methylpyrazolone-5 was added to $25 \mathrm{~mL}$ of 0 -xylene. $4.8 \mathrm{~g}(70 \mathrm{mmol})$ of phosphorus oxychloride was slowly added to the flask. The reaction mixture was then refluxed for 12 hours. After cooling, solvent, side product, and excess phosphorus oxychloride were removed under reduced pressure. A saturated solution containing $20 \mathrm{~g}$ of sodium bicarbonate was added to the flask under an ice bath, and the flask was sonicated. The white precipitate was filtered and extracted with chloroform with water. After washing with water and drying over magnesium sulfate, the solution was filtered and the solvent was removed under reduced pressure to afford the product as a white powder $(0.30 \mathrm{~g}, 25 \%$ yield $) .{ }^{1} \mathrm{H} \mathrm{NMR}\left(500 \mathrm{MHz}, \mathrm{CDCl}_{3}\right)$ : $\delta$ (ppm) $6.33(1 \mathrm{H}, \mathrm{s}), 2.37(3 \mathrm{H}, \mathrm{s}) \cdot{ }^{13} \mathrm{C}$ NMR $\left(500 \mathrm{MHz}, \mathrm{CDCl}_{3}\right): \delta$ (ppm) 14.3, 90.0, 135.5, 153.5. HRMS (APCl+): calculated m/z for C12H12N6 ([M+H]+1): 241.12; found m/z: 241.12 
a.

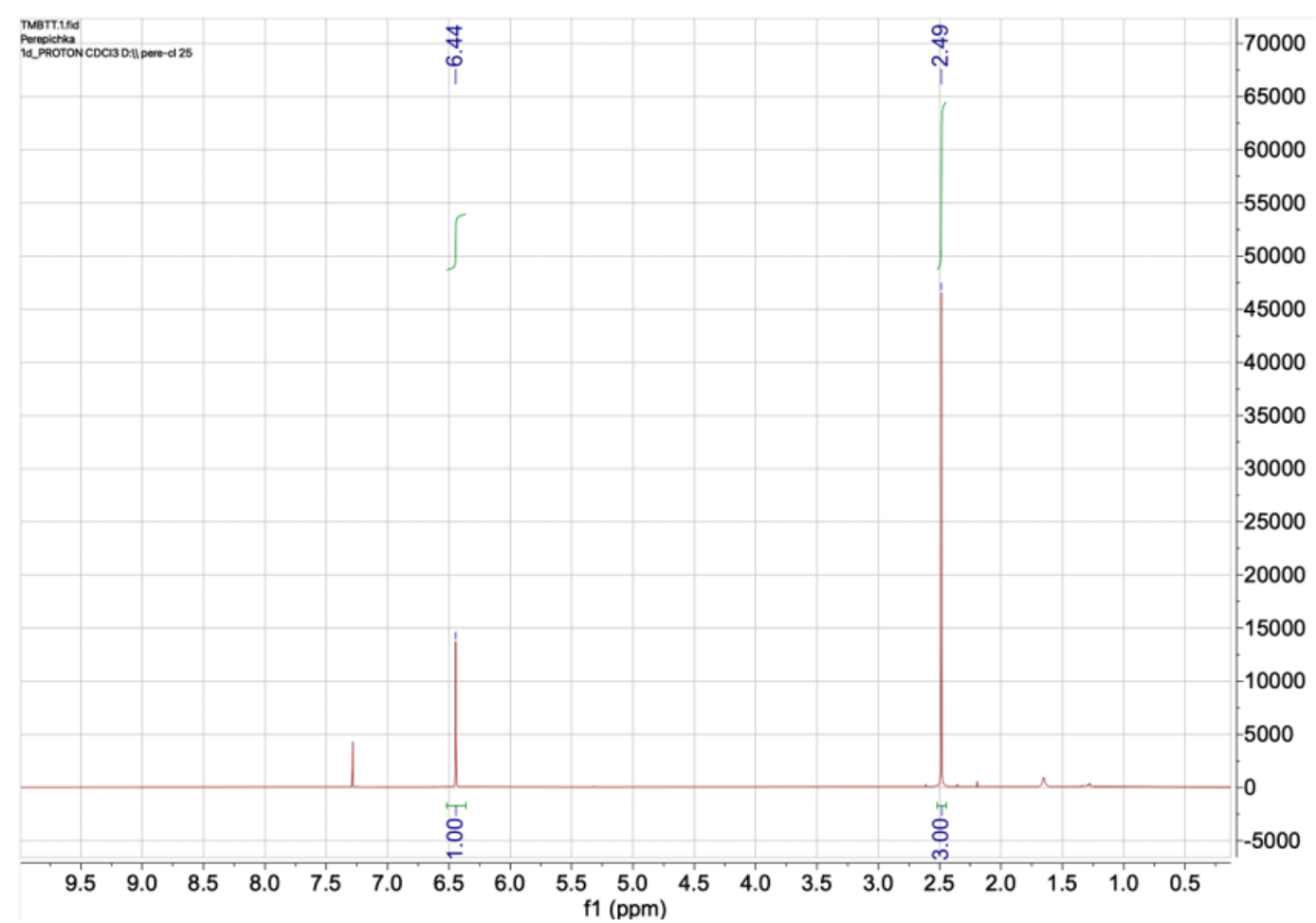

b.

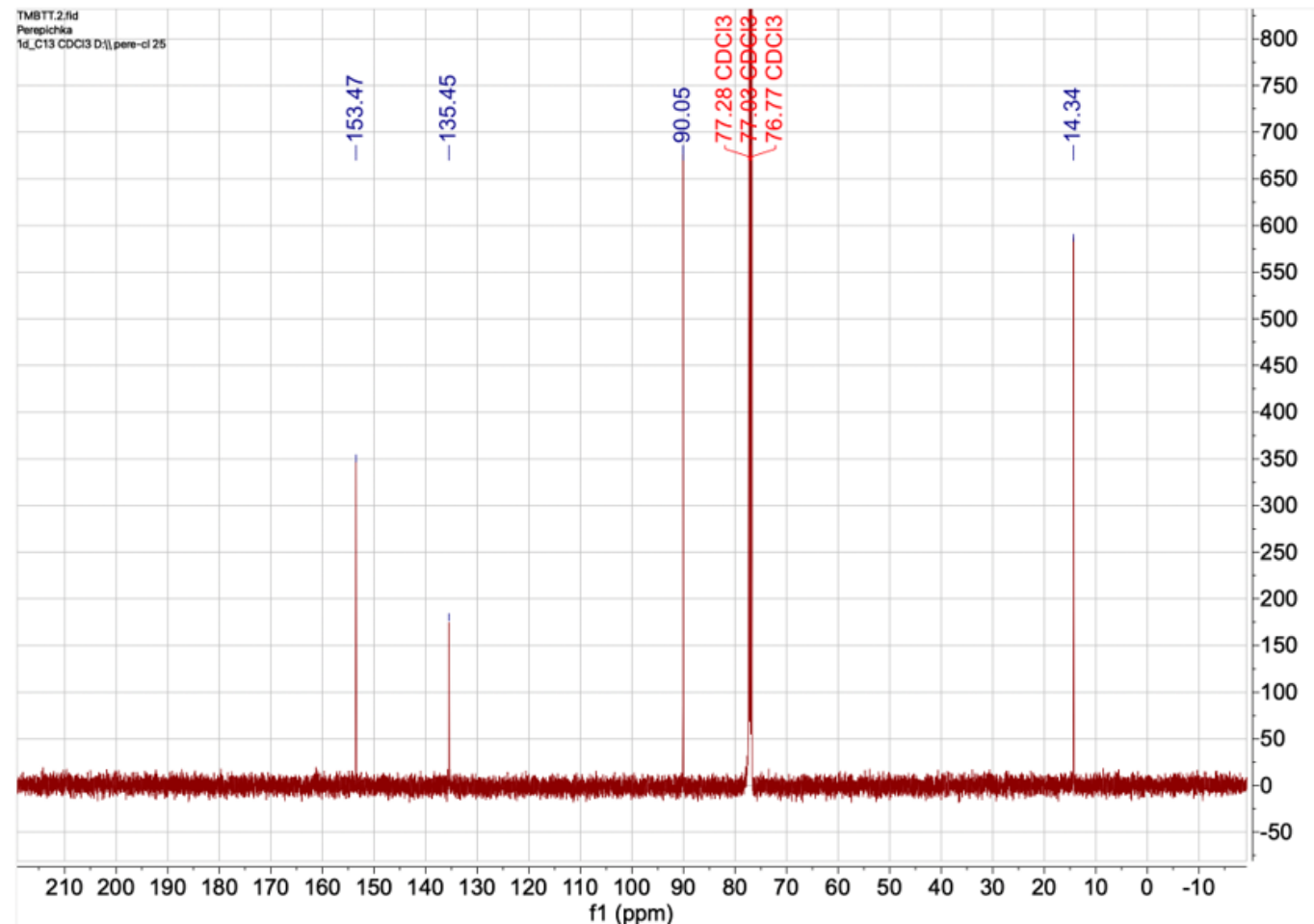

Figure S1. ${ }^{1} \mathrm{H}-\mathrm{NMR}$ (a) and ${ }^{13} \mathrm{C}-\mathrm{NMR}$ of TMTPT(b) 


\section{Additional STM images}
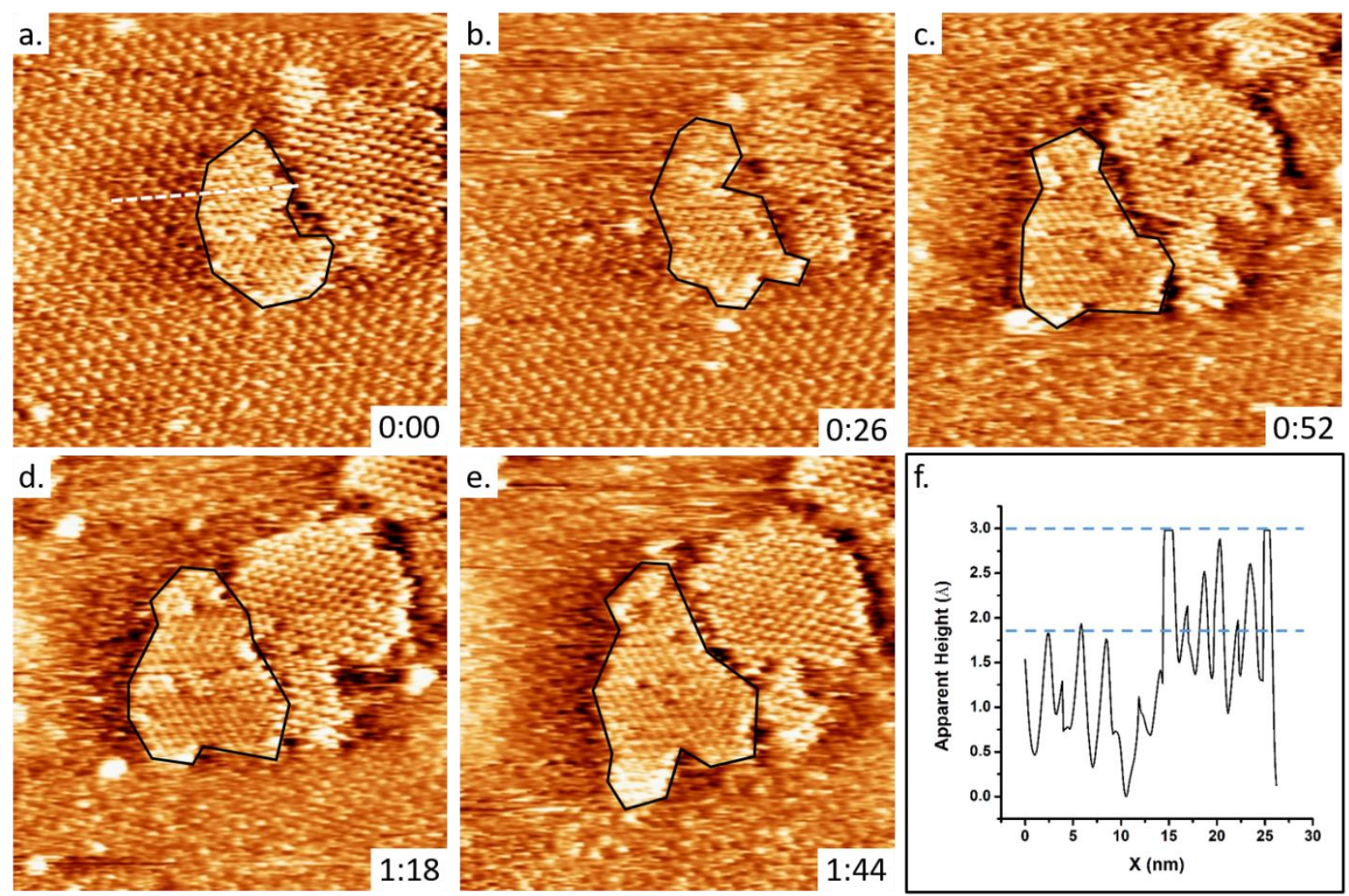

Figure S2. (a-e) STM images showing the gradual transformation from TTBTA monolayer honeycomb to TMTPT-TTBTA dense bilayer phase. In the initial image (a), the dense bilayer packing emerges as brighter small domains surrounded by the monolayer TTBTA honeycomb phase. Cross-section analysis (f) confirms the brighter domain as a dense bilayer structure. Upon continuous scanning of the same region, the transition process from the monolayer honeycomb phase to the dense bilayer phase is revealed. The brighter domain gradually grows up, starting from its domain boundaries as labeled by solid black curves. Image dimensions: $60 \times 60 \mathrm{~nm}^{2}$. Scanning conditions: $\mathrm{U}=-1.5 \mathrm{~V} ; \mathrm{I}=50 \mathrm{pA}$. 


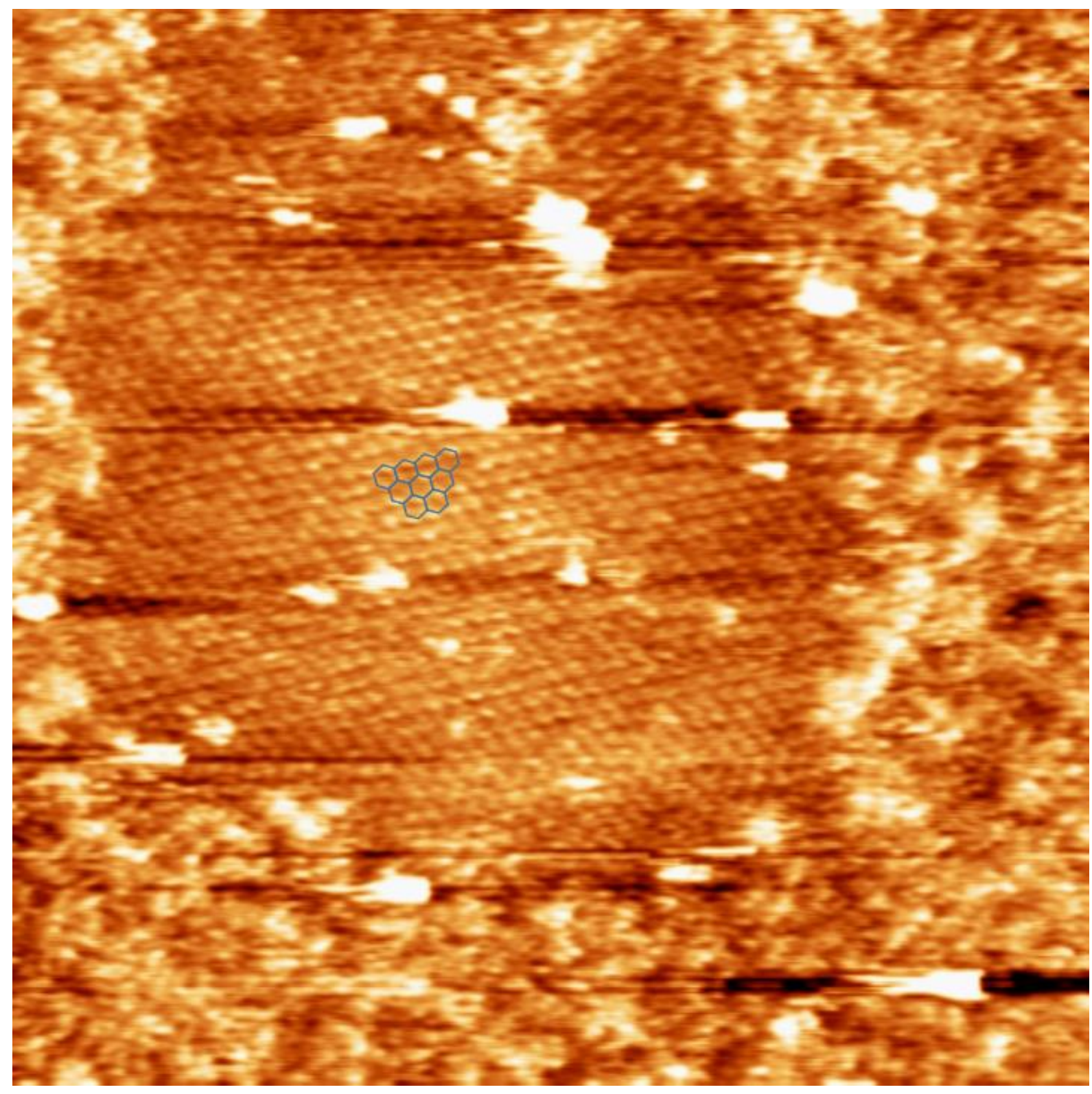

Figure S3. STM image revealing the presence of the honeycomb phase even at a high $\left(5 \times 10^{-4} \mathrm{M}\right)$ concentration of TTBTA. Disordered molecular aggregated on the edges of the images are likely due to precipitation from an over-saturated solution. Image dimensions: $120 \times 120 \mathrm{~nm}^{2}$. Scanning conditions: $\mathrm{U}=-1.5 \mathrm{~V} ; \mathrm{I}=50 \mathrm{pA}$. 

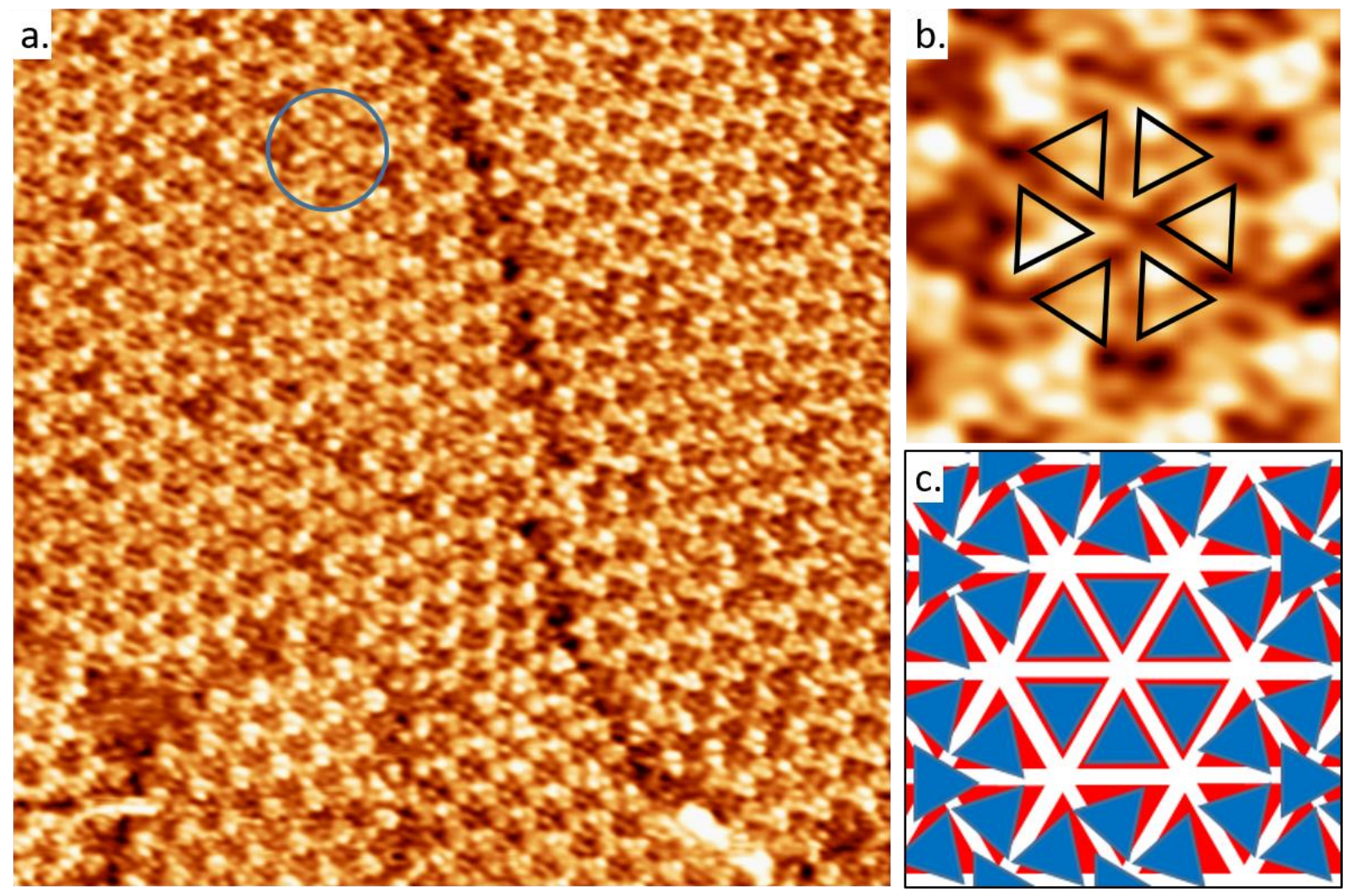

Figure S4. (a) STM image revealing the adsorption of TMTPT molecules on TTBTA dense phase. Image dimensions: $37 \times 37 \mathrm{~nm}^{2}$. Scanning conditions: $U=-1.5 \mathrm{~V} ; \mathrm{I}=50 \mathrm{pA}$. (b) Magnified STM image corresponding to the region indicated by the open blue circle in (a), showing a defective unit cell composed of six eclipsed TTBTA-TMTPT dimers, which disallows the adsorption of the third TMTPT in the unit cell. Image dimensions: $5.5 \times 5.5 \mathrm{~nm}^{2}$. (c) The schematic image of (b). TTBTA and TMTPT are denoted by red and blue triangles, respectively.

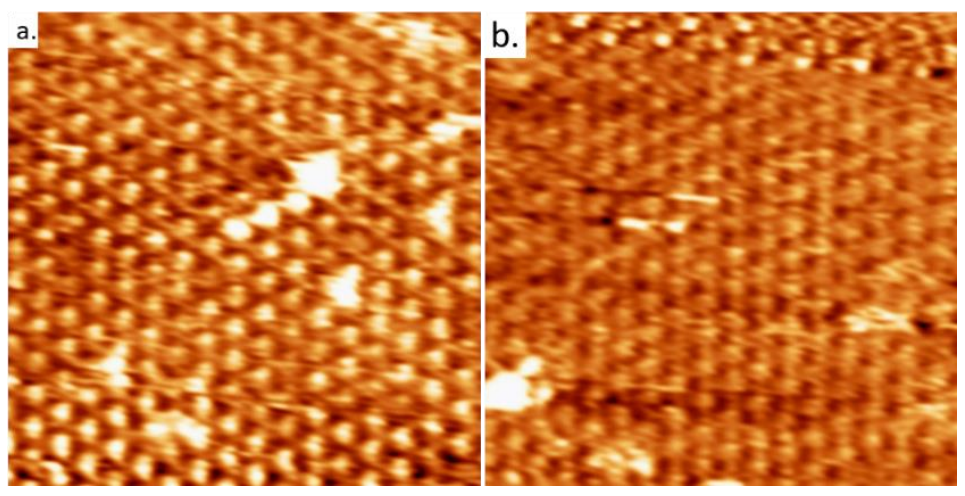

Figure S5. (a) STM image reveals the isolated TMTPT molecules adsorb on top of the TTBTA honeycomb phase. The brighter spots represent the adsorption of TMTPT on TTBTA chicken wire. These features disappear in a consequent scanning (b). Images dimensions: $35 \times 35 \mathrm{~nm}^{2}$. Scanning conditions: $\mathrm{U}=-1.5 \mathrm{~V} ; \mathrm{I}=60 \mathrm{pA}$. 

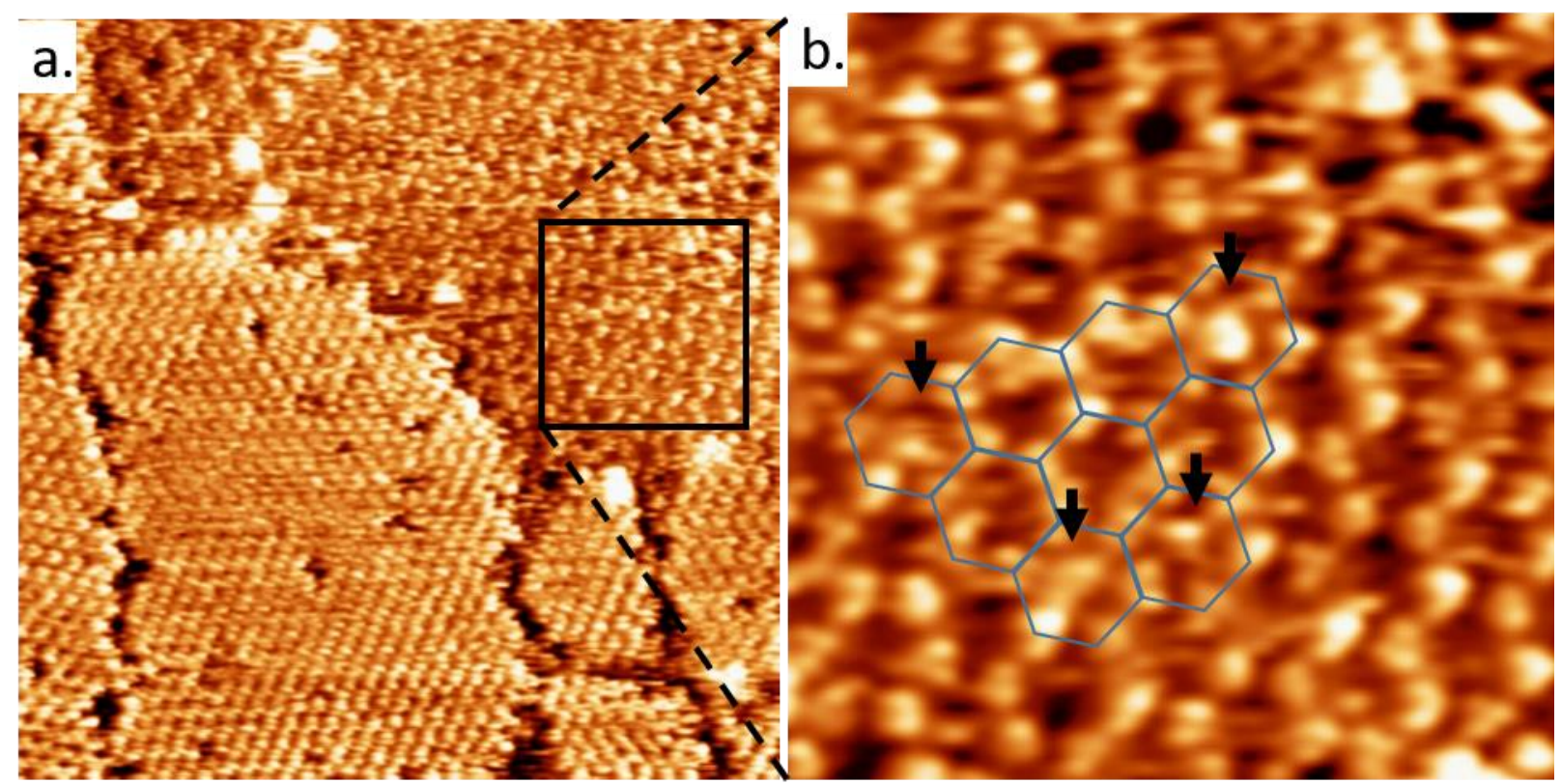

Figure S6. (a) STM image showing the coexistence of monolayer TTBTA honeycomb structure and dense bilayer structure. The sample was prepared by sequentially depositing TTBTA solution and diluted TMTPT solution $\left(10^{-5} \mathrm{M}\right)$. Image dimensions: $60 \times 60 \mathrm{~nm}^{2}$. Tunneling conditions: $U=-$ $1.5 \mathrm{~V} ; \mathrm{I}=50 \mathrm{pA}$. (b) Magnified STM image corresponding to the black open square in (a). Blue hexagonal mesh denotes the honeycomb structure, and the black arrow indicates the occupancy of the pore-site. Image dimensions: $16.6 \times 16.6 \mathrm{~nm}^{2}$.

Despite not forming a bilayer structure with TMTPT, the porous TTBTA honeycomb network can accommodate a molecule to constitute a host-guest structure, as shown in Figure S6. The blue hexagonal mesh represents the honeycomb structure, and the black arrows identify the occupied cavities. Since both TTBTA and TMTPT can adsorb in the pore ${ }^{2}$ we can not distinguish the property of the adsorbed molecule.

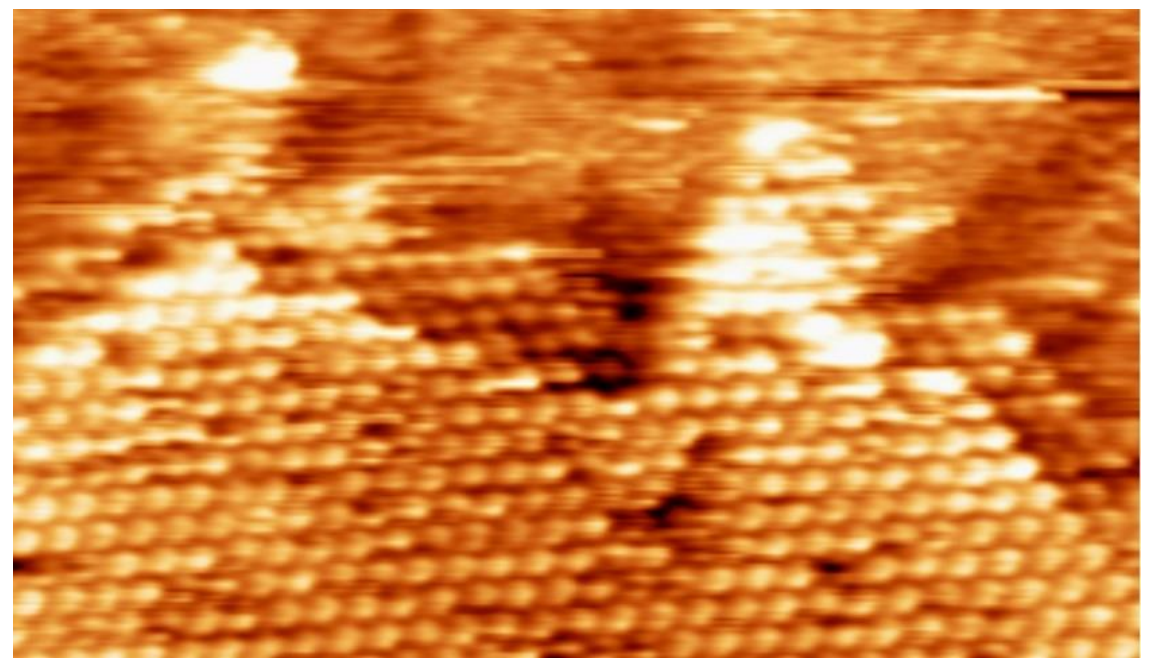

Figure S7. Multiple layer $\mathrm{C}_{60}$ on the honeycomb of TTBTA. ${ }^{3,4}$ Image dimension: $70 \times 40 \mathrm{~nm}^{2}$. Scanning conditions: $U=-1.2 \mathrm{~V} ; \mathrm{I}=30 \mathrm{pA}$. 


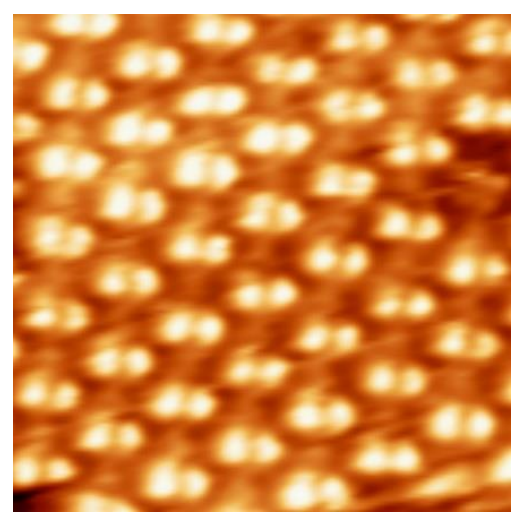

Figure S8. STM image of the sample prepared by sequentially depositing TMTPT and $\mathrm{C}_{60}$ shows fullerene dimers ${ }^{2}$ adsorption arranged in a hexagonal packing. Image dimensions: $15 \times 15 \mathrm{~nm}^{2}$. Scanning conditions: $U=-1 \mathrm{~V} ; \mathrm{I}=40 \mathrm{pA}$.
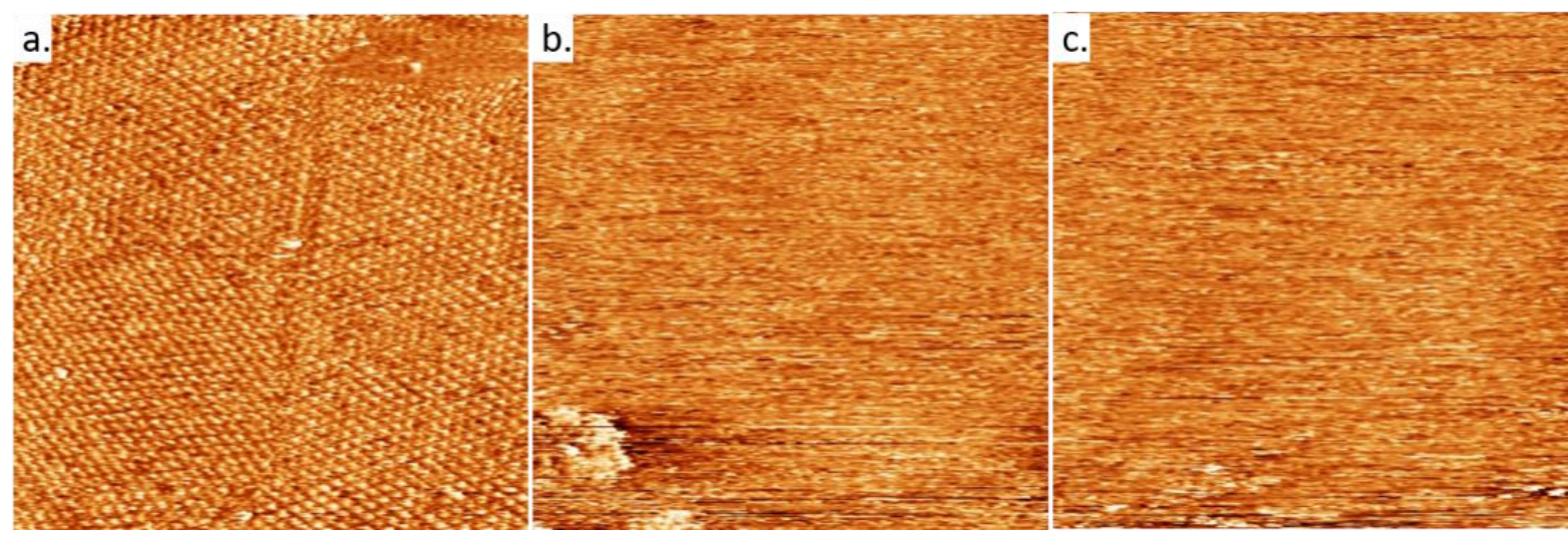

Figure S9. (a) STM image showing the adsorption of $\mathrm{C}_{60}$ in the TTBTA honeycomb phase. Image dimensions: $90 \times 90 \mathrm{~nm}^{2}$. Scanning conditions: $U=-0.9 \mathrm{~V} ; \mathrm{I}=20$ pA. (b,c) Sequential STM images, collected from the sample (a) after adding TMTPT. Images dimensions: $120 \times 120 \mathrm{~nm}^{2}$. Scanning conditions: $\mathrm{U}=-1 \mathrm{~V} ; \mathrm{I}=20 \mathrm{pA}$.
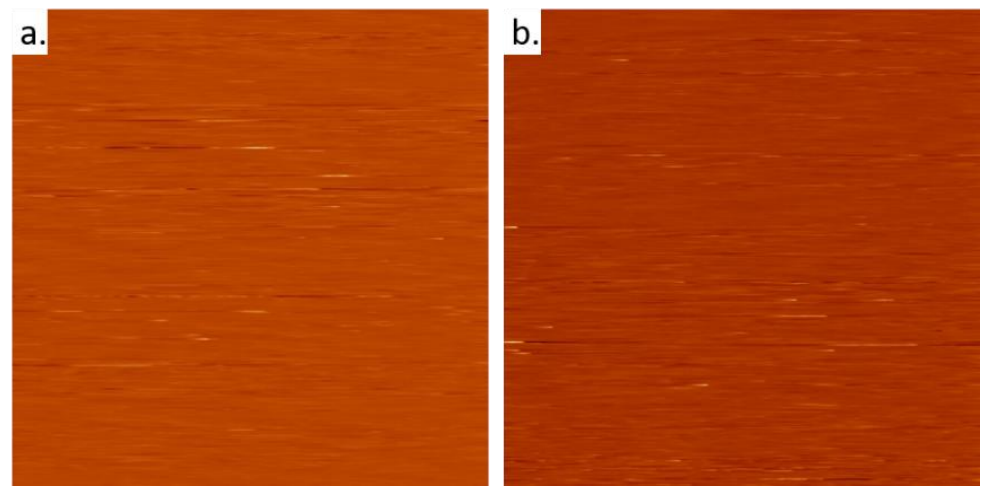

Figure S10. (a) STM image acquired at the interface of TMTPT solution $\left(10^{-3} \mathrm{M}\right)$ and HOPG. No self-assembly was observed. Image dimensions: $60 \times 60 \mathrm{~nm}^{2}$. Scanning conditions: $\mathrm{U}=-1.05 \mathrm{~V}$; I $=50 \mathrm{pA}$. (b) STM image collected from the sample prepared by depositing TMTPT $\left(10^{-3} \mathrm{M}\right)$ and $\mathrm{C}_{60}\left(1.2 \times 10^{-3} \mathrm{M}\right)$ solutions on HOPG. No self-assembly was observed. Image dimensions: $60 \times 60$ $\mathrm{nm}^{2}$. Scanning conditions: $U=-1.2 \mathrm{~V} ; \mathrm{I}=50 \mathrm{pA}$. 


\section{Simulations}

\section{Eclipsed Dimer}

a.

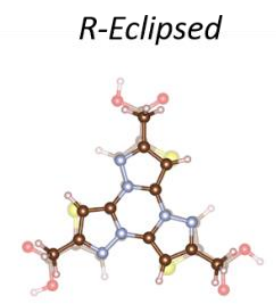

$E=-412.324 \mathrm{eV}$ $\Delta \mathrm{E}=-0.0513 e \mathrm{~V}$ b.

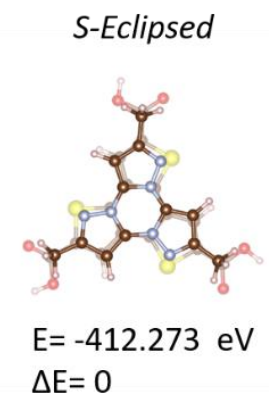

C.

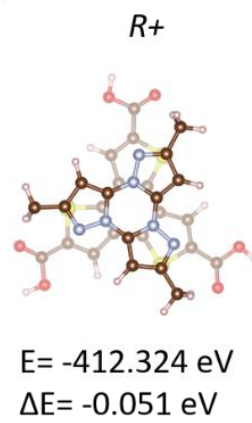

e.

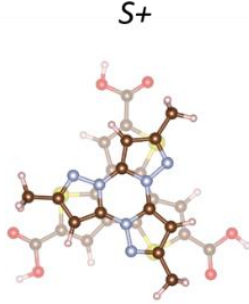

$E=-412.394 \mathrm{eV}$

$\Delta \mathrm{E}=-0.121 \mathrm{eV}$

$\Delta \mathrm{E}_{\text {TTBTA-TTPT }}=-0.776 \mathrm{eV}$ e.

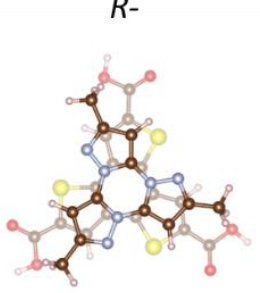

$E=-412.361 \mathrm{eV}$

$\Delta \mathrm{E}=-0.088 \mathrm{eV}$ f.

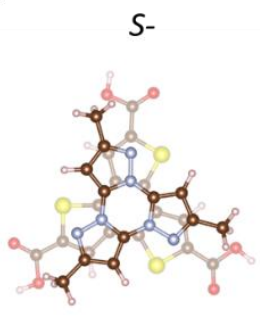

$\mathrm{E}=-412.298 \mathrm{eV}$ $\Delta \mathrm{E}=-0.025 \mathrm{eV}$

Figure S11. DFT optimized TTBTA-TMTPT stacking $\pi$-complex in different configurations. Giving a fixed TTBTA (S) molecule, two eclipsed dimers and four staggered dimers can be created by varying the chirality of TMTPT (R/S) and its rotation relative to the TTBTA (S) molecule. (+ for clockwise and - for counterclockwise) Each architecture can be denoted by combining TMTPT chirality $(\mathrm{R} / \mathrm{S})$ and their relative rotation $(+/-)$. For example, the $S_{+}$dimer represents rotating TMTPT (S) molecule clockwise (+) relative to the given TTBTA. For the eclipsed dimers, we denote them as R-Eclipsed and S-Eclipsed, respectively. The optimized energy is reported in corresponding figures relative to S-Eclipsed, which has the highest energy. The cohesive energy of the $S+$ structure was calculated at $-0.776 \mathrm{eV}$. 
a.

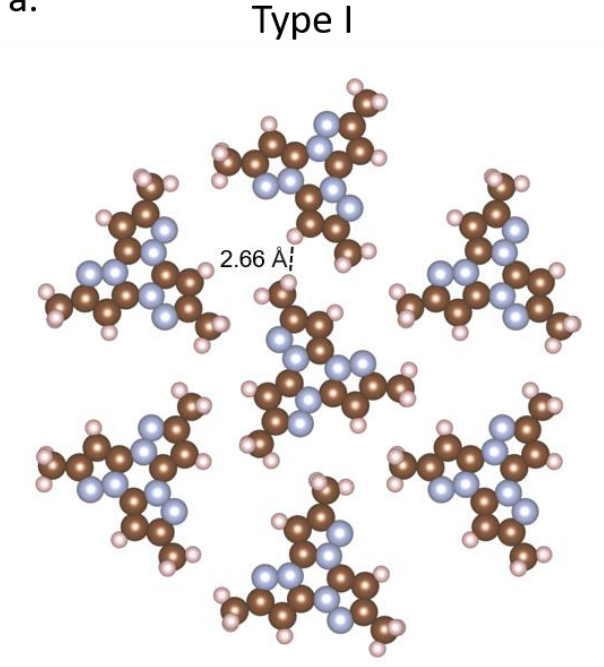

c.

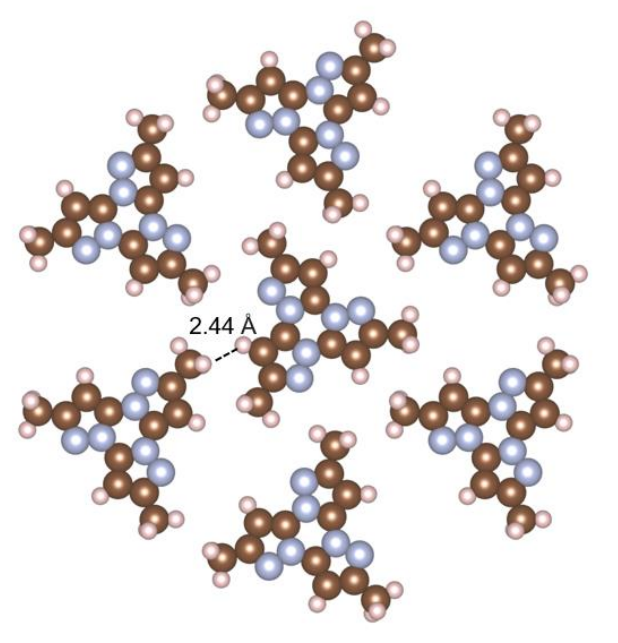

b.
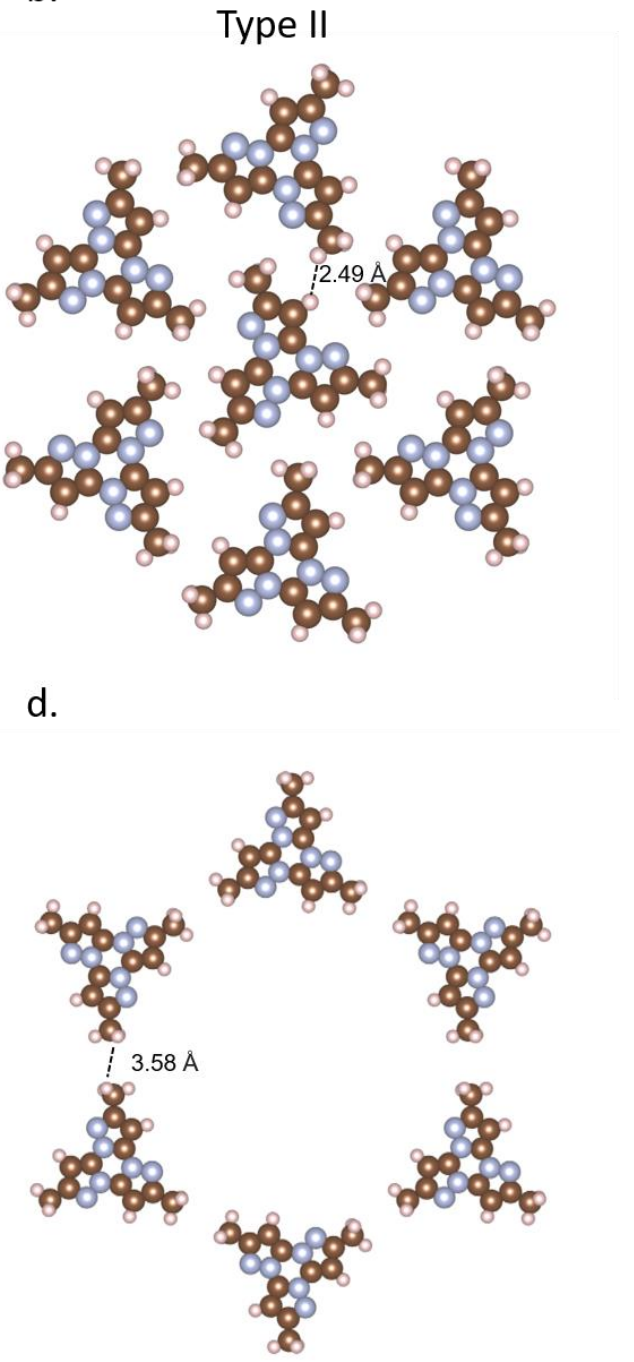

Figure S12. (a-c) DFT optimized structures of TMTPT self-assembly with different chirality when adsorbed on TTBTA dense phase. (d) DFT optimized structure of supposed TMTPT selfassembly on TTBTA honeycomb phase with a constant lattice parameter of $2.4 \mathrm{~nm}$. The distance between the two closest hydrogens is labeled.

\section{References}

1. Taerum, T.; Lukoyanova, O.; Wylie, R. G.; Perepichka, D. F., Synthesis, Polymerization, and Unusual Properties of New Star-Shaped Thiophene Oligomers. Org. Lett. 2009, 11, 3230-3233.

2. MacLeod, J. M.; Ivasenko, O.; Fu, C.; Taerum, T.; Rosei, F.; Perepichka, D. F., Supramolecular Ordering in Oligothiophene- Fullerene Monolayers. J. Am. Chem. Soc. 2009, 131, 16844-16850.

3. Cui, D.; Ebrahimi, M.; Rosei, F.; Macleod, J. M., Control of Fullerene Crystallization from 2D to 3D Through Combined Solvent and Template Effects. J. Am. Chem. Soc. 2017, 139, 16732-16740.

4. Cui, D.; MacLeod, J. M.; Rosei, F., Planar Anchoring of $\mathrm{C}_{70}$ Liquid Crystals Using a Covalent Organic Framework Template. Small 2019, 1903294. 